\title{
Satellite Servicing in Mission Design Studies at the NASA GSFC
}

\author{
Stephen J. Leete \\ Space Science Missions Branch, \\ NASA Goddard Space Flight Center, Greenbelt, MD, USA 20771 \\ 301.286.9093, Stephen.J.Leete@nasa.gov
}

Abstract-Several NASA missions in various stages of development have undergone one-week studies in the National Aeronautics and Space Administration (NASA) Goddard Space Flight Center (GSFC) Integrated Mission Design Center (IMDC), mostly in preparation for proposals. The possible role of satellite servicing has been investigated for several of these missions, applying the lessons learned from Hubble Space Telescope (HST) servicing, taking into account the current state of the art, projecting into the future, and implementing NASA long-range plans, and is presented here. The general benefits and costs of injecting satellite servicing are detailed, including components such as mission timeline, mass, fuel, spacecraft design, risk abatement, life extension, and improved performance. The approach taken in addressing satellite servicing during IMDC studies is presented.

\section{TABLE OF CONTENTS}

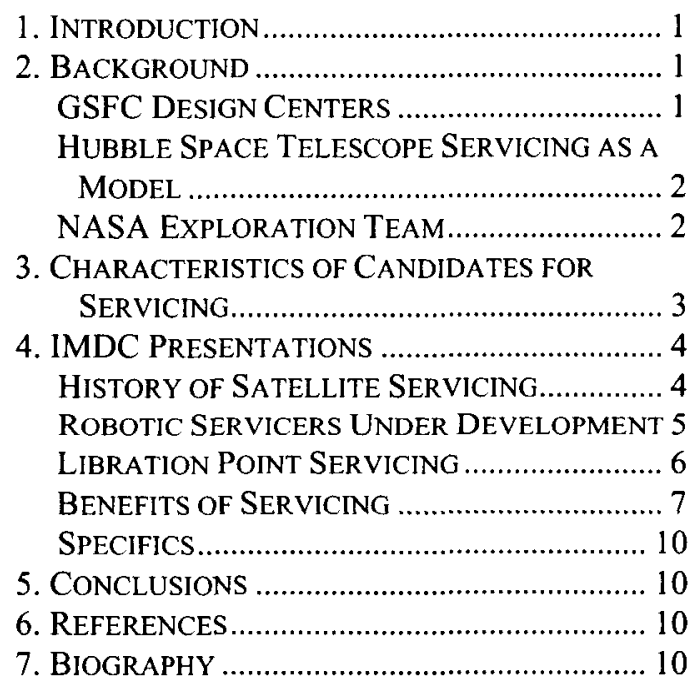

\section{INTRODUCTION}

Since the beginning of humans' entry into space, many capabilities have been developed for sophisticated space operations. Satellite servicing capabilities have been based primarily on astronauts and cosmonauts performing Extravehicular Activity (EVA) with a variety of tools, plus astronauts operating robotic arms from within the shirtsleeve environment of the Space Shuttle crew compartment, all in Low Earth Orbit (LEO). The culmination of this has

\footnotetext{
C) U.S. Government work not protected by U.S. copyright
}

been the servicing of the Hubble Space Telescope (HST) and assembly of the International Space Station (ISS). NASA and others are now looking at taking next steps toward a broader capability to service a variety of missions in an expanded range of orbital locations.

The IMDC has studied several ambitious missions in support of proposals by GSFC principal investigators and others. In June 2002, the author was assigned to be the InSpace Servicing discipline engineer, on staff to the IMDC. The author participated in selected studies for missions that had promise for benefiting from in-space servicing. Because of the competitive nature of these studies, the specific names of the programs studies will not be discussed in this paper. The material provided to IMDC customers, observations by the author and other IMDC participants, lessons learned, and other benefits will be synthesized from the three missions studied into non-sensitive information that can be freely divulged.

\section{BACKGROUND}

\section{GSFC Design Centers}

The Goddard Space Flight Center (GSFC) of the National Aeronautics and Space Administration (NASA), located in Greenbelt, Maryland, has a mission that includes developing space science and earth science missions. Most of the funds spent by NASA on such missions are now awarded to the winners of competitive bids in response to announcements of opportunities. A significant part of the response of the GSFC to this competitive environment was the development of a pair of design centers that are able to quickly bring to bear focused engineering creativity and analysis to support proposals. The design center in which the work presented here was performed is called the Integrated Mission Design Center (IMDC).

The Integrated Mission Design Center (IMDC) is a human and technology resource dedicated to innovation in the development of advanced space mission design concepts to increase scientific value for NASA and its customers. The IMDC and the Instrument Synthesis \& Analysis Laboratory (ISAL) are integral components of Goddard Space Flight Center's Integrated Design Capability (IDC). The IMDC Mission is to develop, define and design mission system concepts to support pre-formulation and formulation phase activities, and leverage engineering expertise to provide an integrated data product in a timely and cost-effective 
manner. The IMDC provides specific engineering analysis and services for mission design, and provides end-to-end mission design products with capabilities that include mission studies, including system/subsystem concepts, requirements, and trades; new technologies \& risk assessments; technical reviews \& focused studies. [1]

\section{Hubble Space Telescope Servicing as a Model}

This investigation of the possible role of servicing for these future missions is an extension of the experience of the GSFC in the servicing of the Hubble Space Telescope (HST). The author has served since 1998 as EVA Systems Engineer, with responsibilities for development of EVA procedures and tools for HST Servicing Missions 3A (STS103, December, 1999), SM3B (STS-109, March, 2002) and SM4 (STS-124, planned for 2004 or 2005). The study of inspace servicing of future missions is consistent with the vision of NASA's future as described in various publications by the NASA Exploration Team (NExT).

NASA recognizes that the Hubble Space Telescope has been an immensely successful mission. It has been highly productive in many ways. When launched in 1990, it produced the first factor-of-ten improvement in angular resolution in the visible range since Galileo first observed the sky with a telescope in 1610. It has provided data for more than 3873 scientific papers. HST has been regularly rejuvenated, using practices that are routine for earth-bound observatories such as adjusting for optical defects, replacing detectors with state of the art devices as they become available through the natural advance of engineering, and upgrading facility equipment such as computer processors and memory, power supplies and pointing control devices. Exercise of these common-sense maintenance and upgrade strategies has resulted in a system which, twelve years after deployment, is still at the cutting edge of technology and scientific utility. Even as its ground-based competitors improve, with adaptive optics to enable Hubble-class angular resolution and ever larger primary optics, HST has been oversubscribed by a consistent factor of 5-6 (which increased to 8 after the SM3B installation of the Advanced Camera for Surveys) as its newer instruments take advantage of its location on-orbit to explore important scientific niches beyond the reach of ground-based systems. [2]

\section{NASA Exploration Team}

The NExT vision includes human participation in missions in orbits beyond low earth orbit (LEO). Many current and future missions launched by the GSFC occupy Lagrange Point (or Libration) orbits. These include the Wind mission (which later was moved into highly elliptical Earth orbits), Solar Heliospheric Observatory (SOHO), and Advanced Composition Explorer (ACE) and Genesis at the Sun-Earth L1 (SEL1) located 1.5 million kilometers from Earth in the direction of the Sun, and the Microwave Anisotropy Probe (MAP) at the Sun-Earth L2 (SEL2) located 1.5 million kilometers from Earth in the direction away from the Sun. SEL2 is also the planned location for GSFC's James Webb Space Telescope. [3]

NASA plans include building a variety of large and complex observatories, made up of either single spacecraft or constellations of spacecraft, which will be located in either Sun-Earth libration orbits (SELx) or other orbits, either at the edge of or beyond the Earth's gravitational influence. [4] There are a number of characteristics of these systems that make them good candidates for in-space servicing. These include high cost, high scientific value, difficulty of ground verification, improved scientific return through incremental improvements, and desire for lengthy operations.

It has been a mantra of NASA, under the leadership of former Administrator Daniel Goldin, to construct missions that were faster, better and cheaper. This philosophy has led to a blossoming of scientific achievement and to a much larger number of missions. Some of the future missions may be accomplished by building multiple copies of relatively small spacecraft that work together in a sophisticated manner as interferometers or stereo imagers or in other creative manners that produce enhanced science in very innovative ways. These constellations will probably be serviced primarily through replacement of individual components with new ones, or launch of entire new constellations. However, if a robust in-space servicing infrastructure is in place, even these systems may benefit from servicing upgrades.

NASA has formulated its overall exploration objectives into three grand challenges. [5] These are presented in Table 1:

Table 1 - Questions and Objectives

\begin{tabular}{|c|c|}
\hline $\begin{array}{c}\text { Grand } \\
\text { Question }\end{array}$ & Exploration Objectives \\
\hline $\begin{array}{l}\text { How did we } \\
\text { get here? }\end{array}$ & $\begin{array}{l}\text { Looking backward in time toward the } \\
\text { early Universe } \\
\text { - Revealing and understanding the } \\
\text { laws of nature } \\
\text { - Determining the role of gravity and } \\
\text { other fundamental processes in the } \\
\text { origin and evolution of life } \\
\text { - Exploring the history of the Solar } \\
\text { System } \\
\text { - Understanding the origin of solar } \\
\text { variability and its effect on Earth } \\
\text { - Exploring the paths of life on the } \\
\text { Earth. }\end{array}$ \\
\hline $\begin{array}{l}\text { Where are } \\
\text { we going? }\end{array}$ & $\begin{array}{l}\text { Understanding the future habitability } \\
\text { and sustainability of Earth } \\
\text { - Expanding human presence beyond } \\
\text { the vicinity of Earth. }\end{array}$ \\
\hline Are we & - Revealing the cycles of life in the \\
\hline
\end{tabular}




\begin{tabular}{|l|l|}
\hline alone? & Universe \\
& $\begin{array}{l}\text { - Searching for life in the Solar System } \\
\text { - Searching for life in the Universe. }\end{array}$ \\
\hline
\end{tabular}

Terrestrial Planet Finder (see Figure 1) is a constellation of large space telescopes that would be capable of directly observing terrestrial planets about another star. Stellar Imager is a similar constellation of imaging space telescopes using a sparse aperture and interferometry to image other stars similar to the way we image our own sun. Other large and complex missions explore other wavelength regimes (such as X-ray), gravity waves, Earth observations, space solar power, etc. [5]

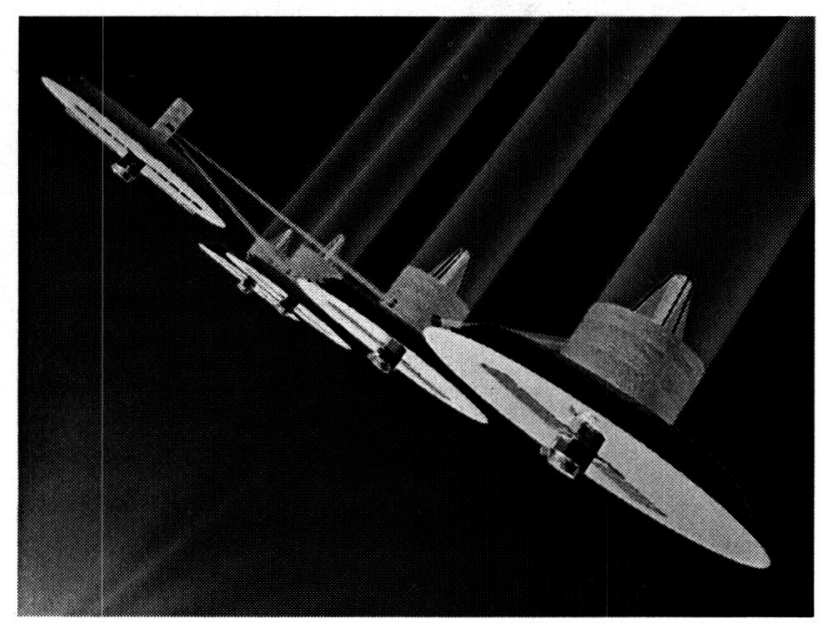

Figure 1 - Terrestrial Planet Finder

\section{Characteristics OF Candidates For SERVICING}

A case has been made to users of the IMDC that some of their systems share many characteristics with HST that make in-space servicing a potential benefit to their mission. For example, some missions studied at the IMDC include precursor missions to grand challenge missions such as Stellar Imager and Terrestrial Planet Finder. Each of the major characteristics of a mission that is a good candidate will be explored further.

High Cost-The HST cost about $\$ 1.5$ billion prior to launch in 1990, and substantially more than that has been spent cumulatively on mission operations and repair missions. In contrast, missions discussed in this paper that were recently studied in the IMDC are mostly precursor, proof of concept missions that are to be done with budgets of a few hundred million dollars, including launch and mission operations. For these missions, it is not possible to include the full cost of a servicing mission in the overall budget of the mission. The only scenario that would likely work in that case is for NASA to decide that it was worth servicing, and to provide the funds for the servicing mission above and beyond the basic mission costs. Development of the servicing spacecraft will probably need to be developed by NASA separately, analogous to NASA's development of the Space Shuttle, used for (among other things) HST servicing. More study is needed to determine how much money would need to be added to the budget of a program to cover the costs of servicing, and to determine how paying for servicing compares with other options, such as building and launching a replacement or upgraded second copy of a mission.

One key consideration will be the cost of a servicing mission. A robotic servicing mission will, in many instances, be less expensive than a human servicing mission. This drives the spacecraft to be designed to be serviced by a relatively simple robotic servicer, such as that proposed for the Orbital Express program. The less serviceable a design, the greater the need for direct human intervention, and the greater the cost of the servicing missions. Designing for servicing up front can, in an environment in which a variety of servicing options is available, result in substantial operational savings.

High Scientific Value-Some of the missions being studied are precursors to future, higher scientific value missions. While these missions will provide some good science value themselves, their primary value will be in allowing scientists and engineers to learn how to do that type of mission, and to take the next step toward the ultimate goal. If the mission is not successful in achieving its goals, and therefore puts the ultimate mission in question, it may be worthwhile to service the precursor mission to the point of achieving mission success so as to keep the overall science mission on track.

Difficulty of Ground Verification-Missions studied at the IMDC have very challenging requirements, sometimes beyond the current state of the art. They can involve interferometric combination of light from multiple telescopes distributed along a truss or on separate freeflying spacecraft. These systems will be extremely difficult to test on the ground. The cost of a pre-launch verification program that has a very high probability of success will be very high. Limited scope proof of concept demonstrations to raise the technology readiness level (TRL) of portions of the mission may help with this. An even better approach may be to quickly and inexpensively put the system together, launch it, test it on-orbit in real conditions, and be prepared to fix it on-orbit once the difficulties are fully understood. This eliminates costly simulation of the space environment, and substitutes 'learning by doing' for 'learning by pretending to do'. The disadvantage is that the problem may not be able to be fully diagnosed remotely, but a well-instrumented system can often be understood. Then servicing features built into the design can be used first to achieve mission success, and later for improvements.

Improved Scientific Return Through Incremental Improvements - One of the hallmarks of HST is the increased scientific productivity over time through upgrades and improvements. Improvements have included optics, detectors, filters, pointing control, power generation, data 
storage, and computation. Some of the missions planned rely on active digital feedback control loops whose bandwidth and overall performance depend strongly on the speed of on-board computing, which in turn is improving rapidly. Science return is also a combination of communications bandwidth and on-board data reduction. Once these missions have been launched and checked out, they will form an in-space infrastructure comparable to HST, that can be incrementally improved upon by adding new technology.

Desire For Lengthy Operations-Once a precursor mission has been launched, it is not clear how quickly funds will become available for the next, more capable mission. It may be desirable to operate even precursor missions for long durations due to overall NASA funding profiles. Servicing could enable this by maintenance, refurbishment and upgrades. Once the "ultimate" version of the mission is put in place, it will be even more desirable to operate it for an extended time, well beyond reasonable design lifetimes of 7-15 years.

\section{IMdC Presentations}

Principal Investigators are provided with a presentation that includes a history of satellite servicing, a summary of robotic servicers under development, future plans for satellite servicing at Libration point orbits, the NASA Exploration Team, benefits of servicing, benefits of initial deployment and checkout at Earth-Moon Libration point orbits, and specifics for how all this applies to their mission. The specifics include various impacts to the mission. These topics are discussed in detail below.

\section{History of Satellite Servicing}

In the late 1970 's and 1980 's, there was a standard design for a satellite that was designed for servicing. This was the Multi-Mission Modular Spacecraft (MMS). See Figure 2 for an exploded view of a typical MMS bus. The MMS was a simple, compact, versatile and robust spacecraft architecture, intended to be applicable to a wide variety of spacecraft missions. Spacecraft of this design were built for solar observation (Solar Max Mission, launched 1980), Earth observation (Landsat 4 and Landsat 5 launched in 1982 and 1984, the Upper Atmosphere Research Satellite (UARS) launched in 1991) and astronomy (Extreme Ultraviolet Explorer (EUVE) launched in 1992). Although specifications for a GEO version were written, no spacecraft of this type were ever built. [3] [6]

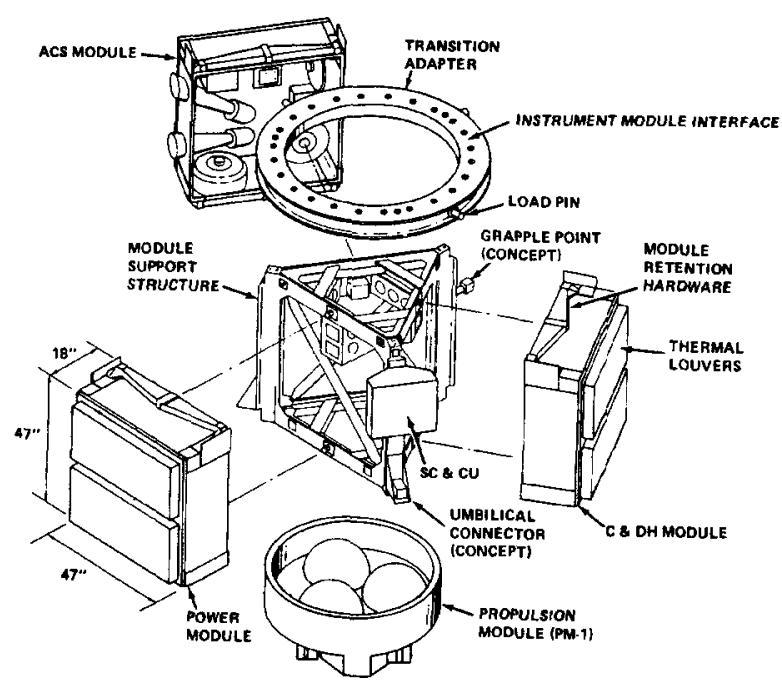

Figure 2 - MMS Spacecraft Bus

The MMS design had many excellent features. The large, externally mounted modules were able to be serviced either by EVA astronauts using simple power or manual hex drive tools or robotically using the Servicing Aid Tool. The spacecraft bus and science payload could be separated robotically in the payload bay. The design included a standard grapple fixture compatible with the Shuttle Remote Manipulator System (RMS). Some of these capabilities were demonstrated in the Solar Max Repair Mission in 1984, the first ever mission to service an un-tended spacecraft (i.e., not a space station). [3]

NASA also demonstrated the ability to retrieve geostationary earth orbit (GEO) communications satellites (comsats) that were stranded in LEO by the recovery of the Westar and Palapa spacecraft in 1984. In 1985, NASA and the Hughes Aircraft Company demonstrated the ability to repair a GEO comsat (Syncom IV) in LEO using an innovative repair kit to electrically bypass an internal relay using test connectors that were accessible to EVA astronauts.

After the Challenger disaster in 1986, NASA stopped routinely using the Space Shuttle for launching commercial satellites, decided not to develop the ability to launch the Space Shuttle from the west coast of the US into polar orbits, and reduced its goals for number of flights per year. All of these decisions impacted the concept of routine servicing of large numbers of spacecraft in a variety of orbits.

After the return to flight of the Space Shuttle fleet, NASA and Hughes Aircraft Company demonstrated the ability to capture a very large spinning GEO comsat, install a new perigee kick motor, and send it off to orbit. These missions showed the versatility of EVA astronauts and astronauts operating the Shuttle RMS, as well as clever designers preparing tools and repair kits, to work on satellites that were not designed for on-orbit servicing. 
Since 1993, the only spacecraft to be serviced in orbit, other than space stations Mir and ISS, is the HST. To date, it has been serviced four times, in 1993, 1997, 1999 and 2002. The HST design does not allow for robotic servicing due to the placement of nearly all components behind doors as well as other details of component design. The design is compatible with EVA servicing, as demonstrated by the very successful series of servicing missions. [3]

A key feature to keep costs down for servicing missions is to enable robotic servicing. The precise implications of this statement for a particular mission are difficult to assess today. There is not an infrastructure of robotic servicers with well-defined capabilities to design around. In order to provide IMDC customers with a sense of current and future capabilities, they are presented with a summary of the current state of the art.

\section{Robotic Servicers Under Development}

Customers are provided with information about the Ranger Robot being developed by the University of Maryland, the Special Purpose Dexterous Manipulator built by MD Robotics of Canada for the ISS, and the Robonaut being built by the NASA Johnson Space Center for ISS, and Orbital Express being developed for the Defense Applied Research Projects Administration (DARPA).

Ranger-Ranger is under development for a Shuttle robotics demonstration on a Spacelab pallet. The development has taken place under the direction of Professor David Akin, founder and head of the Space Systems Laboratory at the University of Maryland, College Park. This particular mission was being funded by NASA, but is not currently funded due to issues with NASA budgets and Space Shuttle manifests, but a significant amount of hardware has already been built for flight. The University of Maryland has a Neutral Buoyancy Research Facility, which is a $15.2 \mathrm{~m}$ diameter by $7.6 \mathrm{~m}$ deep pool used to test Ranger and other space robot concepts under simulated weightless conditions.

Ranger is a pair of 8 degree-of-freedom arms, a body and camera base, a third arm for a "leg", plus a variety of endeffectors to turn bolts, grasp items, etc. Images of a Ranger robotic arm are given in Figure 3 and Figure 4. Ranger typically has two arms, and the Shuttle version also has a third large arm that acts as a leg. It is approximately $150 \%$ of human size. The size of Ranger components (especially diameter) is a result of the specific requirements for the Shuttle demonstration mission. For a different mission, the system could be made much lighter.

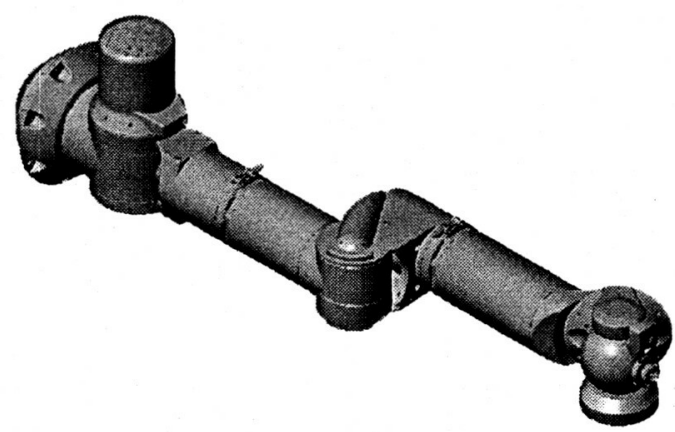

Figure 3 - Ranger Arm Isometric

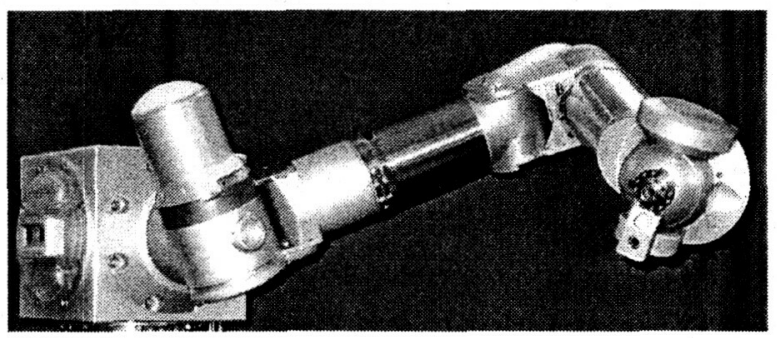

Figure 4 - Ranger Arm Photo

Ranger includes an Interchangeable End-Effector Mechanism that can be built into a variety of end-effectors to allow the end effectors to be stowed and retrieved securely by a Ranger arm without the tether operations that are typical of EVA tool interactions. An example is the Parallel Jaw Mechanism (see Figure 5). Ranger has passed significant milestones toward flight readiness, including safety reviews.

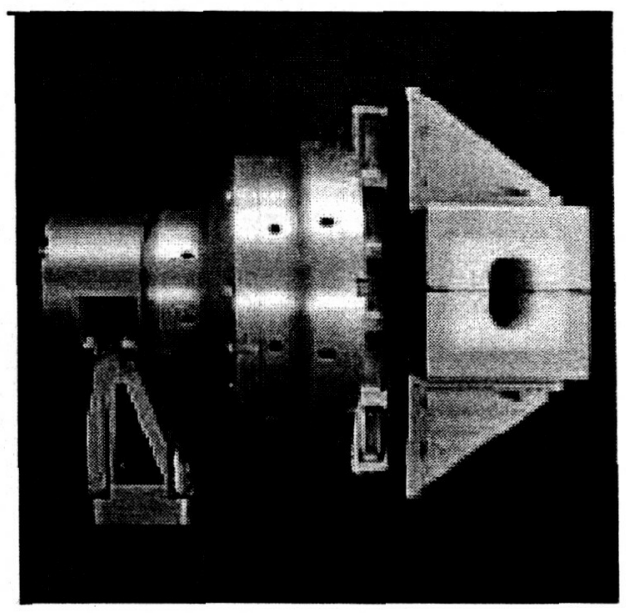

Figure 5 - Parallel Jaw Mechanism

The Ranger concept is to ultimately be a free-flyer satellite servicing system, capable of performing moderately autonomous servicing at a variety of orbital locations, not limited to Shuttle orbits. During development of Ranger, it has focused on performing routine EVA tasks such as HST Orbital Replacement Unit (ORU) change-outs and portable 
foot restraint translation / installation / set-up. It has been explored as an EVA assistant to increase the overall productivity of servicing tasks such as HST servicing and ISS maintenance, as well as a system for end-to-end satellite servicing. Once fully developed, it could be an excellent candidate for servicing NASA missions at Libration point orbits. [7]

Special Purpose Dexterous Manipulator (SPDM)-MD Robotics, using funds from the Canadian government, has built the SPDM for the ISS. It is an important part of the operations concept of the ISS, and is intended to be capable of performing ORU change-outs using only robotics. It extends the capability of the Space Station Remote Manipulator System (SS-RMS) to allow it to interact directly with ORUs that have been designed to be compatible to certain robotic interface standards. The SPDM has been built and tested, and is on the shuttle manifest for installation onto the ISS.

The SPDM is approximately $200 \%$ of human size, with two arms, a grapple fixture for being picked up and translated by the SS-RMS, cameras, lights, and interfaces to a mobile cart. It weighs $1662 \mathrm{Kg}$, can handle ORU's weighing up to $600 \mathrm{~kg}$, is $3.5 \mathrm{~m}$ long, and consumes $600 \mathrm{~W}$ steady state. [8] While its large size is designed for ISS, this may not be suitable for a spacecraft servicer. It should serve as a test bed for this class of robots, and the results should be applicable to smaller, similar systems such as Ranger. The SPDM is shown in Figure 6.

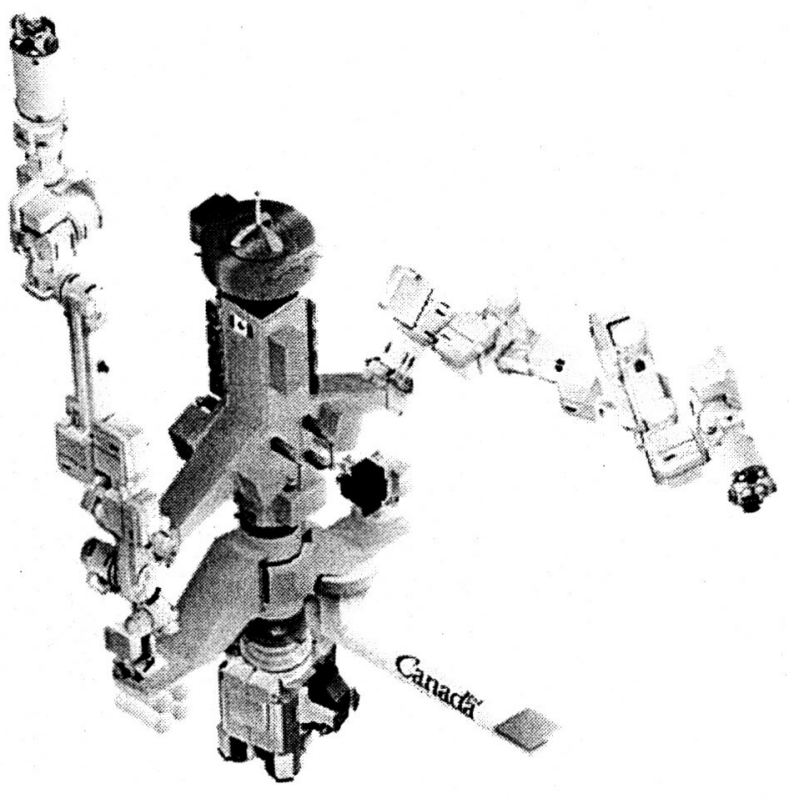

Figure 6 - SPDM

Robonaut-Robonaut is under development at the Johnson Space Center. Both NASA and the DARPA have funded it.
Robonaut is a human-scale, anthropomorphic robot, designed to have capabilities similar to a human in an EVA space suit. Its primary mission is to act as a robotic assistant to an EVA astronaut or cosmonaut on the ISS, to reduce the burden of EVA on the crew. It is capable of using the same EVA tools as the astronauts, and can use the same EVA interfaces on the ISS for translation to work-sites, stabilization at work sites, and acting as a highly dexterous end effector of the SSRMS. Robonaut is shown in Figure 7.

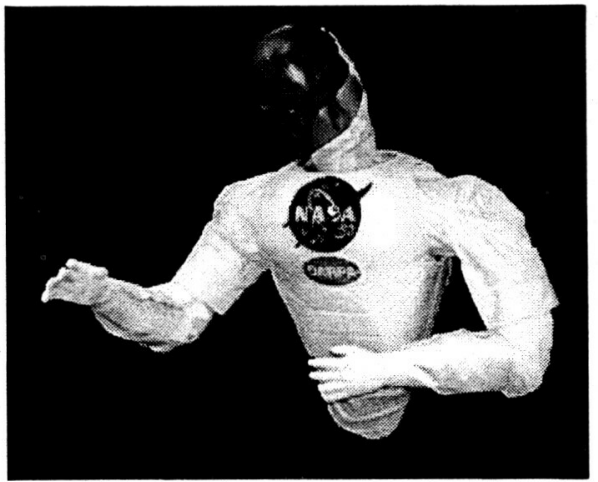

Figure 7 - Robonaut

Orbital Express-Orbital Express is a program funded by DARPA to expand the nation's satellite servicing ability. The objective of Orbital Express is to perform an on-orbit demonstration of autonomous rendezvous and docking, refueling, electrical connection, and Orbital Replacement Unit (ORU) change-out using a single robotic arm. The DARPA assessment is that this type of satellite servicing will be much more cost-effective and more compatible with military operations than shuttle-based human servicing. The US military has at least one future mission that requires onorbit servicing, and Orbital Express will advance the readiness of this key technology. Non-proprietary interfaces for electrical interconnects between spacecraft, ORU mounting and connections, and fluid transfer will be products of the work. The results should also be applicable to commercial and civil space missions. [10]

\section{Libration Point Servicing}

Customers are informed about recent orbital mechanics developments showing that transfers between certain EarthMoon Libration point orbits and Sun-Earth Libration point orb its can be performed with minimal thrusting maneuvers, or very low $\Delta-\mathrm{v}$. For example, the work of Lo [11] and others have described the interplanetary superhighway (see Figure 8 and Figure 9) as a set of related trajectories which form manifolds between families of libration orbits. This work has been applied to servicing scenarios by Joosten [12]. 


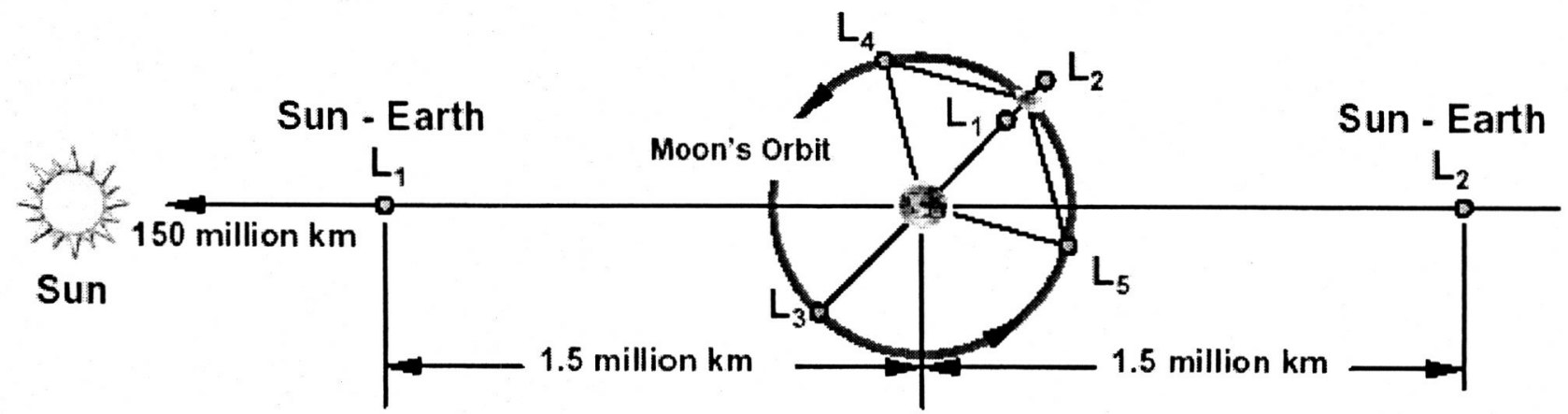

Figure 8 - Libration Point Geometry
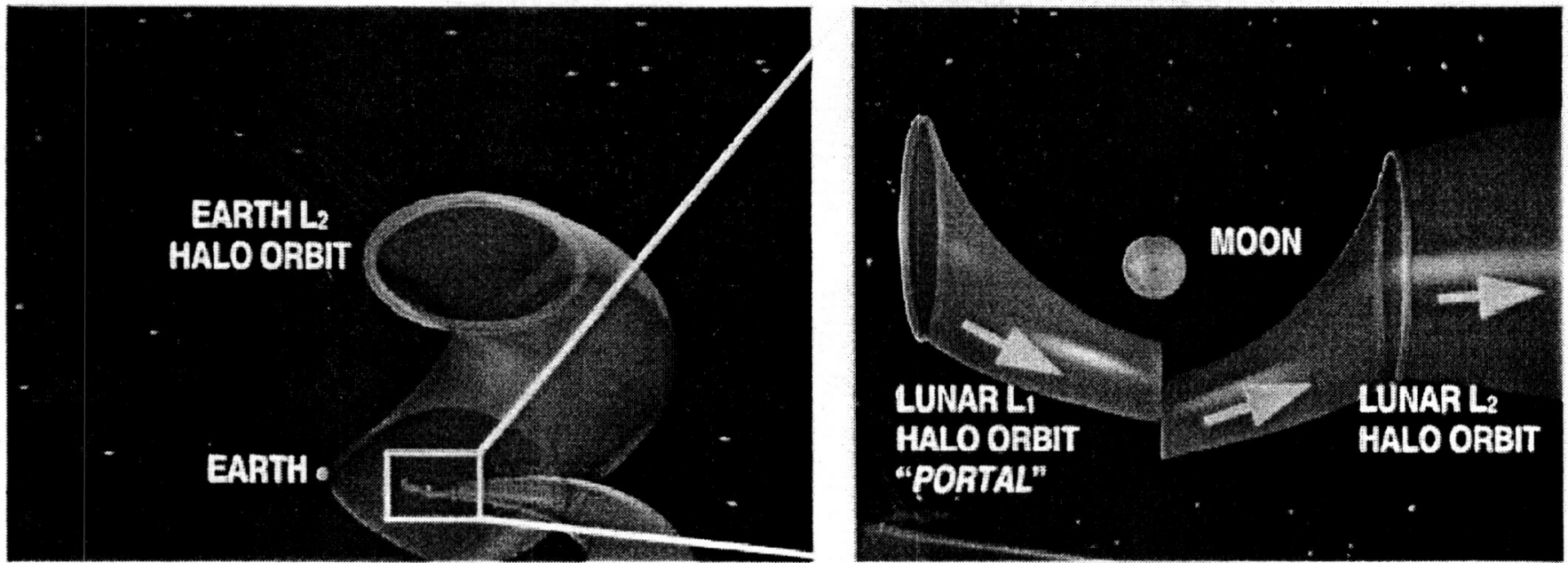

Figure 9 - Lunar L1 Portal to Earth-Sun L2 Halo Orbit

The summary of the NASA Exploration Team plans provided to IMDC customers is a subset of that provided in the background section of this paper.

\section{Benefits of Servicing}

The benefits of servicing can be summarized as fitting within several categories. These will be discussed here as mission success, assembly or assisted deployment, life extension, and capability enhancement. Many of these items were discussed above under characteristics of candidates for servicing.

Mission Success-A spacecraft that has been designed for servicing has the opportunity for initial problems to be corrected at a reasonable cost, short of complete replacement or acceptance of degraded performance. If there is a serious flaw that is found during on-orbit checkout, like the HST mirror or Galileo communications antenna, that significantly impacts mission success, it may be possible to correct it. For example, if a mission is sent on a trajectory to Sun-Earth L2, and a major problem is found, the spacecraft could attempt operations in its operational environment, but after a multiple of 3 or 6 months be put on a trajectory to an Earth-Moon Libration point orbit. By the time it returns to Earth orbit, a repair kit and servicing spacecraft could be put together an positioned for rendezvous. After servicing and initial checkout, the spacecraft is sent back out to its operational orbit. Since many of these missions can continue to make observations during most of the transfer between Sun-Earth L2 and the Earth-Moon system, the scientific down-time can be managed. For a challenging science mission, it is most likely a portion of the science payload that does not perform as expected.

Assembly or Assisted Deployments-The development of large deployable structures that must operate for the first time after launch in zero gravity, vacuum and extreme thermal environments are very challenging. These elements of a mission can be very costly, difficult to test, and are usually mission critical. If these mechanisms can be made to interact with an external agent that can either assemble a deployed structure from pieces, or replace spring and damper systems with a simple hinge and locking bolt scheme, significant savings in development and test can be gained. The cost savings can be estimated by reducing the number of mechanisms which typically cost about $\$ 1 \mathrm{M}$ each to hardware that costs about $10 \%$ of that. Assembly in space also opens up options for much larger sized spacecraft, including spacecraft built up from multiple launches.

Life Extension-A mission that exceeds its design life is likely to begin having a variety of problems, from mechanisms wearing out to radiation degradation of 
electronics. Fuel consumption may also be a limit to operational life. All of these can reliably be resolved by building identical copies of original hardware and replacing it on-orbit. This can result in reduced reliability and life requirements on components, reducing cost and lead time for their procurement, and allowing a wider range of component choices due to lower radiation requirements.

Capability Enhancement-The most exciting possibility for these missions is the ability to renew the system by installing state of the art upgraded replacement components as critical items become obsolete. The example of the Hubble Space Telescope illustrates this very well. The replacement of science instruments on HST has resulted in the correction of the error in the original prescription, larger field of view, higher resolution, better optical throughput efficiency, better dark counts, better quantum efficiency, more pixels, etc. Replacement of tape recorders with solidstate memory improved operations by allowing simultaneous record and playback, increased total memory capacity, improved reliability, and reduced mechanical disturbances. Replacement of the original computer with a 486-based system allowed more complex operations and more on-board processing. Replacement of the solar arrays increased power available, and decreased mechanical disturbances dramatically. The new solar arrays would not have fit in the shuttle for the original launch, and were only able to be added during a servicing/assembly mission.

Perhaps the most interesting work has been the addition of a pair of cooling systems to HST. The NICMOS Cooling System was added on STS-109 in 2002. This functionally replaced a block of solid nitrogen that was part of the NICMOS instrument when it was installed into HST during STS-82 in 1997. After the nitrogen was depleted, NICMOS was no longer able to make observations. The NICMOS Cooling System provided cooling to the NICMOS detectors via a closed-loop refrigeration system, with neon as the working fluid, and the heat rejected to space via flexible capillary pumped loop heat pipes to a large radiator mounted to handrails on the exterior of HST. This has made NICMOS better than new, and able to operate indefinitely. The cryocooler technology is new, and has now been qualified to fly on even the most vibration-sensitive systems for cooling detectors. The Aft Shroud Cooling System, planned to be added to HST on STS-122 in 2004, will relieve thermal limitations on how many instruments can operate simultaneously, increasing the total productivity of HST. The addition of completely new hardware to a system via spacecraft servicing has been demonstrated to be feasible and highly productive. [13]

Costs-Customers of the IMDC are then provided with general and specific information about the costs of servicing. These are generally split into pre-launch costs and costs associated with a servicing mission.

Pre-Launch Costs: Documentation and Configuration Control-Engineers and scientists are capable of amazing creativity and resourcefulness when dealing with a welldefined problem. Three-dimensional computer models, accurate mass properties, metrology of critical interfaces, photographs, drill templates, certification logs, and other sources of insight about a spacecraft that is already on-orbit are all very valuable to the team assigned to perform a servicing mission. Many of these items are good engineering practice for any space mission. However, an additional cost of 1-2 full-time equivalent heads per year during development is a likely impact.

Mass-The mass impact of servicing provisions is a great concern to IMDC customers. The traditional design techniques for enabling servicing involve multiple handrails and foot restraint sockets, grapple fixtures, and other external features. They also include complex latching systems and blind-mate connectors, as well as doors and other atypical features. The design of serviceable satellites is arguably in its infancy. The MMS bus was a first generation, with a payload that could be replaced in its entirety but not repaired on-orbit, although part of the payload was repaired on-orbit. It included bus components arranged in modules that could be replaced on-orbit using a simple robot called the Module Servicing Tool. HST was in some senses a second generation, although in some ways it represents a step backward in design for servicing. While MMS had the potential for robotic servicing, the HST servicing tasks are all complex enough to essentially require humans.

HST servicing mission carriers have typically 5-10 handrails with a mass of $0.5-1.0 \mathrm{~kg}$ each for a total of about $5 \mathrm{~kg}$, and 2-6 Portable Foot Restraint (PFR) sockets with a mass of $0.5-1.0 \mathrm{~kg}$ for a total of about $3 \mathrm{~kg}$ per carrier. Other mass elements include guide rails, blind mate connectors, ORU mounting plates, RMS grapple fixtures, carrier grapple bars, and ORU handles. [14]

An analysis of the mass impacts of varying levels of accommodation for servicing to a variety of spacecraft design is planned as future work in support of IMDC studies. In particular, the author plans to study at least one spacecraft bus that is known to be a current design suitable for libration point orbit missions. This is the Small Explorer (SMEX) - Lite design. This is the bus for the Triana Mission, which has been built and flight qualified, and is awaiting a launch to a Sun-Earth Ll orbit. Modern spacecraft designs have potential for much easier servicing accommodation. For example, many have a simplified electrical architecture of standard data bus and a single voltage for power distribution, greatly reducing the number of electrical interconnects. These were design features of MMS and HST, using earlier technology. There is also the potential for changing out electronics at the card level, launching systems with empty card bays for easy addition of cards, and other approaches which simplify servicing and make it more like maintenance performed on the ground. The author expects to have detailed information on a 
x. serviceable version of at least one spacecraft bus architecture to use for studies by June 2003.

Another standard will be the Orbital Express architecture in support of autonomous robotic servicing. This work is ongoing, but a rough estimate is that the spacecraft to be serviced will have mass impacts of $5 \%-10 \%$ for converting standard components into ORU designs, plus about $50 \mathrm{~kg}$ for the docking and electrical/fluid interfaces module. Because the Orbital Express architecture places a significant part of the burden on the spacecraft being serviced, this can be considered the upper range of mass impacts.

Integration and Test-One consideration is that a spacecraft that has been designed for maintenance on-orbit should also provide benefits during integration and test. However, there are some costs. There will be a need to demonstrate that servicing operations can be performed. Tools that would be used on-orbit should be fit-checked to hardware, although there are ways around this problem if tools are developed after launch. If a robotic system will be used for servicing, then at test should be performed to demonstrate that the robot can do the task. Some of this can be done via 3-D computer modeling with mass properties and dynamics, so that it can be done in parallel with integration and test. Tolerances should be analyzed to show that hardware change-out can be performed even with a temperature differential between the ORU and the spacecraft.

Servicing Mission Costs-Some servicing scenarios include additional maneuvers. If no refueling can be performed onorbit, this needs to be provided for in the fuel budget. If the science mission goes directly to its operational orbit, and only has to transfer between a Sun-Earth L1 or L2 and an Earth-Moon L1 or L2, then the propellant requirements will depend on the size of the operational orbit and servicing orbit. A study was conducted by members of the GSFC Flight Dynamics Analysis Branch (Folta, et al.) and the author for this type of mission. For transfer between large Sun-Earth libration point orbits and large Earth-Moon libration point orbits, the delta- $V$ requirements are dependent on the size of the EM and SE Lx orbits. For transfer from a large EML2 orbit of about $20000 \mathrm{~km}$ by $75000 \mathrm{~km}$ to a large SELl orbit of about $900,000 \mathrm{~km}$, the total delta- $v$ needed is negligible, or $0.18 \mathrm{~m} / \mathrm{s}$. By contrast, to depart from the EML2 point to a smaller SEL2 orbit with an out of plane dimension (Y) of $200,000 \mathrm{~km}$, the delta- $\mathrm{v}$ to depart EML2 is $180 \mathrm{~m} / \mathrm{s}$. A typical maneuver into or out of the Sun-Earth L1 or L2 is about $1 \mathrm{~m} / \mathrm{s}$. A maneuver into or out of a more typical Earth-Moon L1 or L2 is about $70 \mathrm{~m} / \mathrm{s}$. [15]

The major cost in terms of propellant is to enable initial assembly and checkout in an Earth-Moon libration point orbit. There is a penalty for inserting first into E-M Ll as opposed to direct transfer from LEO to S-E L1 or L2. This EML1 insertion delta-v is roughly $425 \mathrm{~m} / \mathrm{s}$ for a $27,000 \mathrm{~km}$ by $70,000 \mathrm{~km}$ EMLl orbit. The injection from LEO starts with a delta-v from a $186 \mathrm{~km}, 23.5^{\circ}$ inclination circular orbit and a delta-v of $3.14 \mathrm{~km} / \mathrm{s}$. From this EMLl orbit, a transfer to a SEL1 orbit of 300,00 by $800,000 \mathrm{~km}$ takes a total of 0.2 $\mathrm{m} / \mathrm{s}$, and a transfer time of about 120 days. [15]

Servicing Hardware--If servicing simply consists of refueling, then no ORU hardware is needed unless an entire fuel tank is being changed out. Replacement ORUs will need to be built and tested. If the spacecraft is one in a series of identical copies, then new hardware could be fit-checked into whichever unit is on the production floor at the time. Once there are no more flight units on the floor, it will be highly desirable to have a high-fidelity mockup available, in a clean room in most cases, for fit checks. A copy of the flight avionics may be needed as a test bed. However, with modern systems operating on standard buses, it is much easier to meet this requirement with the same simulators that are used to perform bench testing of the components.

For a science instrument that is to be serviced, the needs become more complicated. In that case, an engineering model of the instrument should be maintained in a flightlike configuration on the ground. This can be used to trouble-shoot any problems that develop on-orbit, and to test solutions to those problems. It also acts as a mock-up for fitchecks and integration testing of replacement hardware, and to practice change-out or add-on servicing operations. While it can be kept in storage while not needed, there will need to be lab space for the troubleshooting and integration activities.

For HST servicing missions performed by astronauts, simulated weightless engineering evaluations and crew training are a critical element. For robotic servicing, a similar approach may be similarly valuable. However, there is a significant difference. For human EVA servicing, the crew needs to be in the suit handling the simulated hardware. For a robotic mission, the human is always in a shirt-sleeve environment, either in space or on the ground. Theoretically, the robot operator could do all of his or her training in a virtual reality simulation, as the robotic arm operators do in preparation for a shuttle servicing mission. However, these simulations do not typically simulate the contact forces, latch operations, or other critical details. It will likely remain useful to have a simulation of the robot and the flight hardware with high fidelity versions of latches and other interfaces, for additional training. For heavy items, under-water neutral buoyancy training will still have a place. For this, versions of the robot and other hardware that can operate under water and be made neutrally buoyant will be needed. Time will need to be procured at an underwater facility that can support these operations, including the time of scuba divers who can set up and support.

Mission Timeline - Once a spacecraft is on a trajectory to a Sun-Earth libration point or drift-away orbit, it may be quite time-consuming to bring it back to a servicing location at Earth-Moon L1 or similar orbit. Conversely, there are. difficulties with assembling large, fragile spacecraft in LEO and thrusting them out to their operational orbit. A low 
thrust mission from LEO will incur significant radiation damage from slow passage through the Van Allen belts. High thrust maneuvers from LEO pose the risk of damage due to loads, especially for deployed or assembled structures. A better solution is to quickly get out of the Earth's gravity well to an Earth-Moon libration orbit, perform deployments, assisted assembly, system check-out, and if necessary an initial set of repairs while still in Earth orbit. However, there is a more general trade space between thrust level, thruster efficiency, radiation belt transit time, radiation hardness, and mechanical delicacy of the system.

\section{Specifics}

For each IMDC study in which in-space servicing is considered, the PI is provided details on how good a candidate his mission would be for servicing, what role servicing could play, what impacts he would have, and what benefits he could expect. Because of the competitive nature of these studies, these specifics will not be presented here. As in-space servicing is considered for more studies, the level of detail is improved and the interaction with other disciplines is enhanced.

\section{Conclusions}

Information about the NASA GSFC IMDC has been provided. Background information about NASA plans in regard to future satellite servicing as part of the NASA Exploration Team findings has been summarized. The information provided to IMDC customers, who are typically principal investigators for science missions for which proposals are being prepared, has been provided. The general benefits and impacts of in-space servicing have been presented. The earlier that decisions can be made to modify the design to accommodate servicing, the less the cost should be and the greater the benefits. The goal of this work is to raise awareness of the appropriate role of in-space servicing on NASA missions at the grass-roots level of principal investigators at the pre-proposal stage of development, and to date this effort has been successful. It remains to be seen when the first proposal that incorporates servicing will be submitted.

\section{REFERENCES}

[1] GSFC IMDC Web Site: http://imdc.nasa.gov/

[2] Hubble Space Telescope 19992000 Biennial Report, NP-2000-8-077-GSFC, Government Printing Office, 2000.

[3] Stephen J. Leete, "Design for On-Orbit Spacecraft Servicing", in Proceedings of 2001 Core Technologies for Space Conference, November 6-10, 2001

[4] National Aeronautics and Space Administration, Strategic Plan 2000, Washington, DC, 2000.

[5] Report of the NASA Exploration Team, "Setting a Course for Space Exploration in the $21^{\text {st }}$ Century", NASA,
June 1999 through December 2000.

[6] Multimission Modular Spacecraft (MMS) External Interface Specification and User's Guide, S-700-11, NASA Goddard Space Flight Center, 9/15/81

[7] Gardell Gefke. Ranger Telerobotic Shuttle Experiment. http://rtsX.ssl.umd.edu/, May 2001. UMD SSL

[8] http://www.mdrobotics.ca/wwdframe.html

[9] Robert Ambrose, Christopher Culbert, \& Fredrick Rehnmark, "An Experimental Investigation of Dexterous Robots Using EVA Tools and Interfaces", American Institute of Aeronautics and Astronautics, AIAA 2001-4593, Albuquerque, 2001.

[10] "Orbital Express Space Operations Architecture / . ASTRO" in http://www.darpa.mil/tto/programs/astro.html

[11] Martin Lo \& S. Ross, "The Lunar L1 Gateway: Portal to the Stars and Beyond", American Institute of Aeronautics and Astronautics, AIAA 2001-4768, Albuquerque, 2001.

[12] B. Kent Joosten, "Exploration in the Earth's Neighborhood Architecture Analysis", July 11, 2000.

[13] Buddy Nelson, et al., Hubble Space Telescope Servicing Mission 3B Media Reference Guide, Lockheed Martin / NASA, 2002

[14] Damon Cali, HST Servicing Mission 3B Mass Properties Report, Orbital Sciences Corporation / NASA, March 2000.

[15] David Folta, et al., "Servicing and Deployment of National Resources in Sun-Earth Libration Point Orbits", 2002 World Space Congress, Houston, TX, October 10-19, 2002.

\section{BIOGRAPHY}

Stephen J. Leete received his BSE in 1981 from Princeton University in Mechanical and Aerospace Engineering, and his MS in 1997 from George Washington University in Mechanical Engineering. At NASA, since 1987 he has focused on spacecraft systems, science instrument systems, and EVA satellite servicing. A member of AIAA, he has been the Instrument Systems Engineer for various GSFC

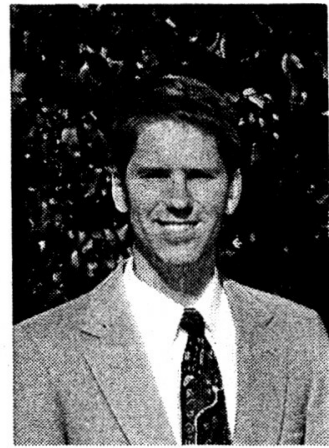
science instruments, and is currently an EVA Systems Engineer for the servicing missions to the Hubble Space Telescope. Mr. Leete resides in Beltsville, MD, with his wife and two young children. 